\title{
Siderophore production and utilization by milk spoilage Pseudomonas species
}

\author{
A. G. Brown ${ }^{1}$ and R. K. J. Luke \\ Department of Agricultural Sciences, School of Life Sciences, La Trobe University, 3086, Australia
}

\begin{abstract}
Many bacteria respond to potentially growth-limiting availability of iron by producing low-molecular-weight iron chelators (siderophores). The aim of this work was to examine the siderophores synthesized and utilized by Pseudomonas spp. implicated in milk spoilage. Twenty isolates of Pseudomonas spp. previously shown to have significant milk spoilage potential were tested for the ability to produce siderophores. Of these, 14 produced pyoverdin and 2 of these also produced pyochelin; 1 produced only pyochelin; 1 produced only salicylate; 2 produced non-pyoverdin, hydroxamate-containing siderophore; and 2 produced chrome azurol sulfonate reactive material that was neither pyoverdin nor pyochelin. There was considerable diversity among the pyoverdins produced. All isolates were shown to utilize iron complexed with exogenous pyoverdin, but usage of particular exogenous pyoverdins differed among isolates. Interference with the iron-uptake systems of the Pseudomonas spp. may be a means by which food spoilage can be slowed, and the pyoverdin system would appear to be a potential target. However, given the diversity of pyoverdins produced and utilized, and the presence of other siderophores, successful interference with bacterial iron acquisition in this context may be challenging.
\end{abstract}

Key words: Pseudomonas, milk, siderophore, iron

\section{INTRODUCTION}

Deterioration in the quality of refrigerated milk is brought about primarily by the action of Pseudomonas spp. (Bishop and White, 1986; Craven, 1993). Historically, Pseudomonas fluorescens has been considered the most important species (Bishop and White, 1986; Craven, 1993), but a recent publication discounts the role of $P$. fluorescens and suggests Pseudomonas fragi and Pseudomonas lundensis may be the most important

Received May 19, 2009.

Accepted December 16, 2009.

${ }^{1}$ Corresponding author: andrew.brown@mgc.com.au species (Marchand et al., 2009). Deterioration follows the release and action of proteases and lipases, and results include off-tastes, lower yields of cheese, and wastage of product (Champagne et al., 1994).

Pseudomonas spp. are aerobic organisms that require iron for growth and development (Neilands, 1973). In an aerobic environment, iron is effectively insoluble at neutral $\mathrm{pH}$ and bacteria must employ specific ironacquisition strategies (Braun et al., 1998). In milk the presence of iron-binding proteins, in particular lactoferrin, further increases the challenge for such bacteria. Siderophore-mediated iron uptake is a strategy that increases both solubility and availability of iron (Neilands, 1973). Siderophores are synthesized inside the bacterial cell and are then excreted into the culture medium, where they bind and solubilize iron. The ferric-siderophore complex enters the cell via an appropriate iron-repressible outer membrane protein receptor (Neilands, 1973). In other bacterial genera blocking of siderophore-mediated entry of iron by means of antibody that is specific for iron-repressible outer membrane proteins has been shown to inhibit growth (Lin et al., 1998). The shelf life and quality of dairy products may be improved by this means if antibodies targeted specifically against iron-repressible outer membrane proteins of appropriate Pseudomonas spp. were to be produced and used to slow the increase in cell numbers. However, given that Pseudomonas spp. protease, lipase, and siderophore are increased in an iron-deficient environment (McKellar et al., 1987; Fernandez et al., 1988) via iron starvation extracytoplasmic sigma factors (Woods et al., 2001), food spoilage may inadvertently be increased.

Of the Pseudomonas spp. implicated in milk spoilage, P. lundensis and Pseudomonas gessardi have been shown to produce pyoverdin (Meyer and Geoffroy, 2004) but production of secondary siderophores by these organisms appears not to have been studied. Pseudomonas fluorescens has been reported in the literature to produce pyoverdin (Meyer and Abdullah, 1978), pyochelin, pseudomonine, quinolobactin, and salicylate (Meyer and Abdullah, 1978), whereas P. fragi has been reported not to produce a siderophore (Champomier-Vergès et al., 1996). Aerobactin, ferrioxamine, corrugatin, and 
2,3-dihydroxy- $N$-benzoyl-arginine are also produced by Pseudomonas spp. but much less frequently (Cornelis and Matthijs, 2002). Pyridine-2,6-dithiocarboxylic acid has also been suggested as a putative siderophore, but this has yet to be confirmed (Leach and Lewis, 2006). Some strains may acquire iron from exogenously derived siderophores, heme, or citrate (Létoffé et al., 1998; Cornelis and Matthijs, 2002). A single strain of Pseudomonas spp. may synthesize more than one siderophore, and the most appropriate siderophore(s) for a bacterium may depend on its immediate environment (Létoffé et al., 1998).

This report describes an investigation of siderophore production and utilization by 20 milk spoilage Pseudomonas spp. (MSP) previously shown to be strongly proteolytic and lipolytic (Craven, 1993).

\section{MATERIALS AND METHODS}

\section{Bacterial Isolates}

Bacterial isolates were obtained from H. M. Craven (Food Science Australia, Werribee, Victoria, Australia). Isolates B52, AFT29, and AFT36 have been described previously (Stehaniak et al., 1982; Dring and Fox, 1983; McKellar et al., 1987). Pseudomonas spp. isolates 3, $13,38,65,71,79,102,107,113,117,328,329$, and 345 were isolated from Victorian milk (Craven, 1993). Pseudomonas spp. isolates RO13 and RO93 were independently isolated from raw Victorian milk (H. M. Craven, Food Science Australia, Werribee, Victoria, Australia; personal communication). All have been phenotypically identified to species level, but recent publications have cast doubt over such identifications (Marchand et al., 2009).

\section{Growth Conditions}

The growth media used were Medium 56 (Greenwood and Luke, 1976) supplemented with $34 \mathrm{~m} M$ sodium succinate; Modified Medium 9 (Schwyn and Neilands, 1987) supplemented with $30 \mathrm{~m} M$ D-glucose and $0.2 \%$ (wt/vol) casamino acids; and casamino acids (Sokol, 1986) buffered with $100 \mathrm{~m} M$ Tris.HCl (pH 7.4). Unless stated otherwise all media were deferrated by means of overnight incubation with Chelex-100 resin (Bio-Rad, Hercules, CA). Iron-sufficient cultures contained 100 $\mu M$ added $\mathrm{FeCl}_{3} \cdot 6 \mathrm{H}_{2} \mathrm{O}$. Solid media were solidified by the addition of $1 \%$ (wt/vol) purified agar (Oxoid, Basingstoke, UK).

All cultures were grown for $48 \mathrm{~h}$ at $25^{\circ} \mathrm{C}$. Unless otherwise indicated the medium used was deferrated Medium 56 supplemented with succinate. The inocu- lum $\left(40 \mu \mathrm{L}, 5 \times 10^{5}\right.$ cells $)$ was made iron deficient by 2 prior 24-h passages in the medium to be used in the subsequent experiment unless stated otherwise. Liquid cultures $(20 \mathrm{~mL})$ were incubated in $250-\mathrm{mL}$ Erlenmeyer flasks shaken at $180 \mathrm{rpm}$ in an orbital incubator and cells removed by centrifugation at $20,000 \times g$ for 20 min at $4^{\circ} \mathrm{C}$.

\section{Nonspecific Detection of Siderophores}

Siderophore production was detected by growing bacteria on Tris-buffered deferricasamino acids agar overlaying chrome azurol sulfonate (CAS) agar that contained only mixtures A and B (Schwyn and Neilands, 1987). Hydroxamate-containing siderophores were detected by means of a modified Czáky assay (Gillam et al., 1981). The Arnow assay (Arnow, 1937) was used to measure catechol-containing siderophores in the cell-free culture supernatant of nonfluorescent isolates grown in Modified Medium 9 supplemented with 30 $\mathrm{m} M$ D-glucose and $0.2 \%$ (wt/vol) casamino acids.

\section{Detection of Pyoverdin, Pyochelin, and Salicylate}

Pyoverdins were detected in culture supernatant by means of fluorimetry (Visca et al., 1992). Pyochelin and salicylate were extracted from acidified culture supernatant with ethyl acetate (Visca et al., 1993). The ethyl acetate was rotary evaporated to near dryness, the concentrate was applied to silica gel 60 thin layer chromatography plates (Merck, Darmstadt, Germany), and the chromatograms were developed in chloroformacetic acid-ethanol (90:5:2.5) (Cox and Graham, 1979). Pyochelin and salicylate were detected by exposure of the thin layer chromatography plate to UV light or spray containing $0.1 \mathrm{M} \mathrm{FeCl}_{3} \cdot 6 \mathrm{H}_{2} \mathrm{O}$ in $0.1 \mathrm{M} \mathrm{HCl}(\mathrm{Cox}$ and Graham, 1979). Pyochelin was also detected by spraying a second thin layer chromatography plate with ammoniacal silver nitrate (Cox and Graham, 1979).

\section{Purification of Pyoverdins from Culture Supernatant}

Pyoverdins used in assays for utilization of exogenous pyoverdin-bound iron were purified from iron-deficient culture supernatant by means of Amberlite XAD-4 resin (Sigma, St. Louis, MO; Meyer et al., 1998). Before HPLC and MS, ferripyoverdins were extracted from culture supernatant by chloroform/phenol extraction (Meyer and Abdullah, 1978). Concentrations of both deferri- and ferripyoverdins were determined by means of spectroscopy, assuming an extinction of $20,000 \mathrm{~cm}^{-1}$ $M^{-1}$ at $403 \mathrm{~nm}$ (Meyer and Abdullah, 1978). 


\section{Fractionation of Extracts Containing Non-Pyoverdin, Hydroxamate-Containing Siderophore(s)}

Solid-phase extraction was used to extract non-pyoverdin, hydroxamate-containing siderophores (NPHS) from the culture supernatant of isolates AFT29 and AFT36. Alltech Sample Prep C18 35-75U (3 g; Alltech Australia, Baulkham Hills, Australia) was placed in a sintered glass funnel. A water pump was used to draw liquid through the column under vacuum, into a vacuum flask.

The extraction protocol involved conditioning the column with $25 \mathrm{~mL}$ of methanol, washing the column with $25 \mathrm{~mL}$ of distilled water, applying $50 \mathrm{~mL}$ of culture supernatant, washing the column with $25 \mathrm{~mL}$ of distilled water, draining residual water from the column with vacuum, and then eluting bound compounds with $25 \mathrm{~mL}$ of methanol. The siderophore-containing methanol was collected, concentrated by rotary evaporation, and freeze-dried.

\section{HPLC and MS}

Extracts containing ferripyoverdins or NPHS were fractionated by HPLC with a Vydac C18 218TP54 column (Vydac, Hesperia, CA). The methodology used for the separation of ferripyoverdins was identical to that of Kilz et al. (1999). A similar method was used to separate the NPHS, but the column was rinsed with $100 \% 50 \mathrm{~m} M$ acetic acid before the commencement of the methanol gradient. The ferripyoverdin fractions with the greatest absorbance (405 nm) and NPHS fractions with any liquid CAS activity were analyzed by means of electrospray ionization MS with a PerkinElmer API-300 triple quadrupole mass spectrometer.

\section{Exogenous Pyoverdin-Bound Iron Utilization Assays}

Utilization of iron bound to exogenous pyoverdins was tested in undeferrated Medium 56 plates supplemented with $34 \mathrm{~m} M$ sodium succinate and a growthinhibitory concentration of deferrated ethylenediamine di(o-hydroxyphenylacetic) acid (appropriate concentration determined for each isolate). The ethylenediamine di(o-hydroxyphenylacetic) acid (Sigma) was deferrated as described by Payne (1994). The plates were seeded with $10^{6} \mathrm{cfu} / \mathrm{mL}$ iron-sufficient cells, previously grown for $24 \mathrm{~h}$ in nutrient broth (Oxoid) and washed in deferrated PBS. Pyoverdin $(30 \mu \mathrm{L}, 1 \mathrm{mM})$ was added to a well cut into the plate that was then incubated for 24 $\mathrm{h}$ at $25^{\circ} \mathrm{C}$ before being examined for the presence of haloes of growth.

\section{RESULTS}

\section{Siderophore Production by the MSP Isolates}

The colonies produced by the $20 \mathrm{MSP}$ isolates under investigation were examined following incubation at $25^{\circ} \mathrm{C}$ for $48 \mathrm{~h}$ on Tris-buffered deferricasamino acids agar overlaid on CAS agar. The colonies produced by 19 of the 20 isolates were surrounded by yellow haloes as would be expected if they were siderophore producers. Colonies produced by the 20th isolate, ATCC 31732, were not surrounded by yellow haloes at $48 \mathrm{~h}$, but were if incubation was prolonged for up to about $14 \mathrm{~d}$.

When the modified Czáky assay was applied to culture supernatants of the $20 \mathrm{MSP}$, significantly higher $(P<0.01)$ levels of hydroxamate activity were detected in iron-deficient cultures than in the iron-sufficient cultures for 16 of the isolates (Figure 1).

Three isolates (RO13, 79 and 329) were found to produce both pyochelin-like material and salicylate-like material, and one (ATCC 31732) produced only the latter (Figure 2). Culture supernatants of 14 of the 20 MSP tested were found to contain material with a fluorescence spectrum that corresponded to that of pyoverdin (Table 1).

The Arnow assay was applied to culture supernatants from the 6 non-pyoverdin-producing isolates. The apparent catechol concentration did not exceed $3 \mu M$ and none produced significantly more catechol-like material in iron-deficient cultures than in iron-sufficient cultures ( $t$-test of 3 replicates per isolate, $P=0.14$ to 0.91 ).

Presumed siderophore production by the 20 MSP is summarized in Table 2.

\section{Heterogeneity of the Pyoverdins}

Ferripyoverdins isolated from culture supernatant were investigated by HPLC and MS. Values for the mass of each deferripyoverdin moiety determined following subtraction of the mass of the attached iron atom were as shown in Table 1. A wide range of molecular masses is evident. Only 3 molecular masses were associated with more than one isolate: 1,098 Da (isolates 38 and 71); 1,352 Da (isolates ATCC 948 and 3), and 1,241 Da (isolates RO93 and 79). The molecular mass of the deferripyoverdin produced by isolate 329 was less than $1 \mathrm{Da}$ greater than the deferripyoverdin(s) produced by isolates RO93 and 3.

\section{Non-Pyoverdin, Hydroxamate-Containing Siderophore(s)}

The NPHS were partially purified from the cell-free culture supernatant of AFT29 and AFT36 by reversed 
phase solid-phase extraction and further purified by reversed phase HPLC. Each HPLC fraction was evaporated to remove methanol and the presence of siderophore-like compound(s) examined by CAS assay. Three fractions from each isolate contained CAS-reactive material and were analyzed further by MS. Five of the fractions were insufficiently concentrated or contained too much salt for MS analysis, but one fraction from AFT36 yielded a compound with a molecular mass of $678 \pm 0.3$ Da.

\section{Utilization of Exogenous Pyoverdin-Bound Iron}

The addition of exogenous pyoverdins reversed ethylenediamine $\operatorname{di}(o$-hydroxyphenylacetic) acid-induced iron limitation of growth of all isolates, although there was significant variation between individual isolates and pyoverdins (Table 3). For example, isolates B52 and RO93 used only 4 exogenous pyoverdins, whereas isolates 71 and 345 used 13 exogenous pyoverdins. Moreover, some pyoverdins stimulated growth of more isolates than did others. For example, whereas isolate 3 pyoverdin was found to stimulate the growth of only 4 other isolates, isolate B52 pyoverdin was able to stimulate the growth of all 19 of the other isolates tested. Growth of Pseudomonas spp. isolates that were not pyoverdin producers could also be stimulated by pyoverdins.

\section{DISCUSSION}

Spoilage of refrigerated milk products is commonly associated with psychrotrophic Pseudomonas spp. Until recently $P$. fluorescens was considered the most important species (Craven, 1993), but a recent publication claims that the role of $P$. fluorescens has been overstated and that P. fragi and P. lundensis are the most important (Marchand et al., 2009). Although not the dominant microbes found in fresh milk and unable to survive pasteurization, the psychrotrophic MSP commonly come to dominate the bacterial population at the time of spoilage because they have the greatest growth rate at refrigerated temperatures (Matselis and Roussis, 1998). The effects of MSP-induced spoilage of milk typically include changes in texture and flavor that result from the action of extracellular proteases and lipases (Suhren, 1989).

The mechanisms used by Pseudomonas spp. to acquire iron have been well studied (for review, see Cornelis and Matthijs, 2002), but much less is known about their role and significance in milk spoilage. In this paper, our focus is on further characterization of the iron uptake mechanisms available to MSP and their potential relevance to the spoilage of milk.

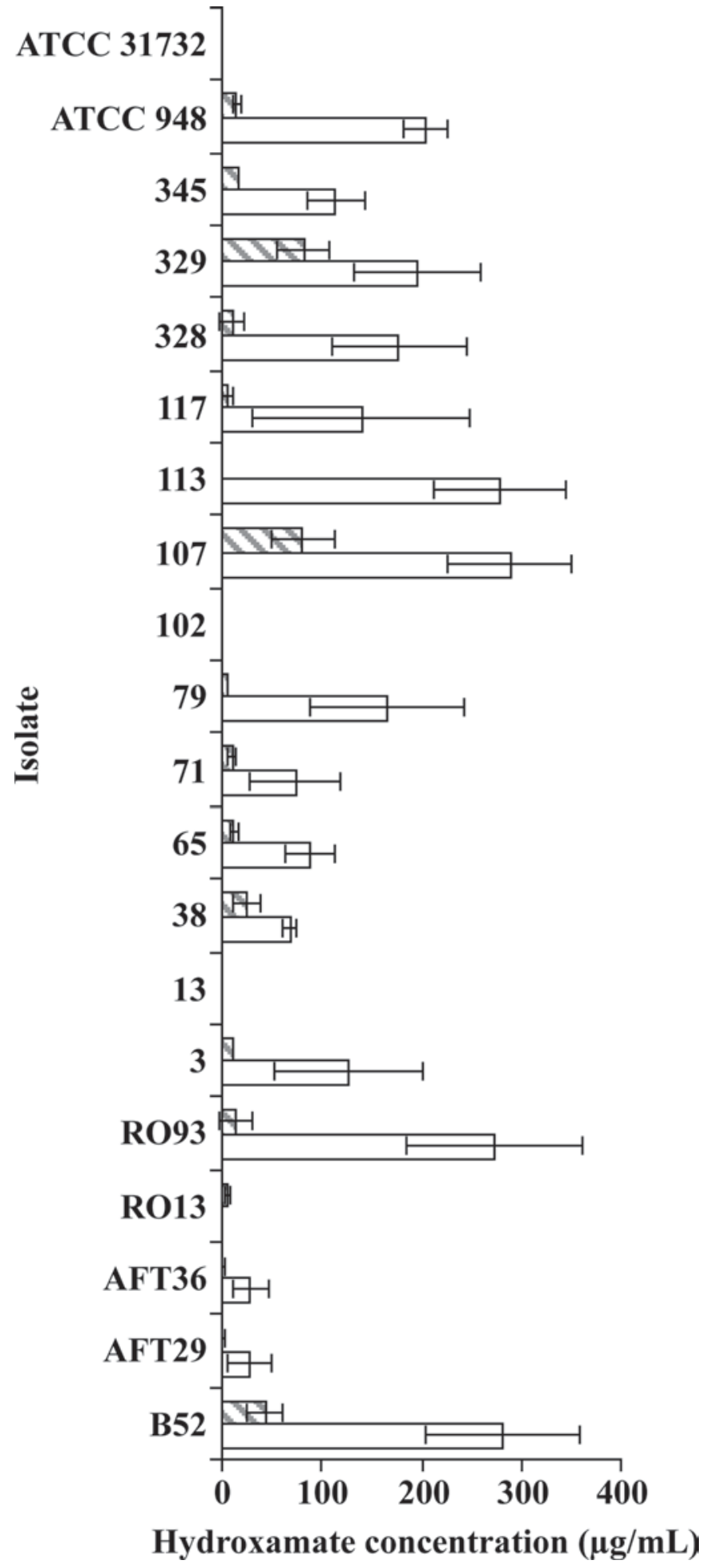

Figure 1. Comparison of the hydroxamate content (modified Czáky assay) in iron-deficient (open bars) and iron-sufficient $\left(100 \mu M \mathrm{FeCl}_{3}\right.$; hatched bars) culture supernatant from milk spoilage Pseudomonas spp. Error bars indicate 3 standard deviations from the mean based on 3 replicates and represent the $99 \%$ CI. 
Table 1. The pyoverdin concentration in iron-deficient cell-free culture supernatant and the HPLC column retention times and estimated molecular masses of the corresponding deferripyoverdins produced by pyoverdinsynthesizing milk spoilage Pseudomonas spp.

\begin{tabular}{lccr}
\hline Isolate & $\begin{array}{c}\text { Pyoverdin } \\
\text { concentration }(\mu M)^{1}\end{array}$ & $\begin{array}{c}\text { Column retention } \\
\text { period }(\min )\end{array}$ & $\begin{array}{c}\text { Estimated molecular } \\
\text { mass }^{2}(\mathrm{Da})\end{array}$ \\
\hline B52 & $271 \pm 10$ & 12 & $1,359.1 \pm 0.8$ \\
RO93 & $356 \pm 20$ & 15 & $1,241.7^{\mathrm{A}} \pm 0.6$ \\
3 & $556 \pm 46$ & 12 & $1,352.8^{\mathrm{C}} \pm 0.4$ \\
38 & $118 \pm 11$ & 12 & $1,098.5^{\mathrm{B}} \pm 0.4$ \\
65 & $127 \pm 13$ & 11 & $1,322.8 \pm 0.6$ \\
71 & $90 \pm 6$ & 9 & $1,098.8^{\mathrm{B}} \pm 0.6$ \\
71 & & 11 & $1,098.8^{\mathrm{B}} \pm 0.6$ \\
79 & $135 \pm 5$ & 15 & $1,241.8^{\mathrm{A}} \pm 0.6$ \\
107 & $239 \pm 20$ & 12 & $1,287.8 \pm 0.5$ \\
113 & $264 \pm 8$ & 15 & $1,472.7 \pm 0.4$ \\
113 & & 12 & $1,444.7 \pm 0.4$ \\
117 & $221 \pm 21$ & 11 & $1,239.7 \pm 0.4$ \\
328 & $217 \pm 18$ & 11 & $1,324.9 \pm 0.4$ \\
329 & $167 \pm 7$ & 14 & $1,242.1^{\mathrm{A}} \pm 0.4$ \\
345 & $410 \pm 25$ & 10 & $1,130.8 \pm 0.6$ \\
ATCC 948 & $158 \pm 7$ & 12 & $1,352.6^{\mathrm{C}} \pm 0.4$ \\
ATCC 948 & & 10 & $1,352.6^{\mathrm{C}} \pm 0.4$ \\
\hline
\end{tabular}

${ }^{\mathrm{A}-\mathrm{C}}$ Values with common letters indicate pyoverdins of identical molecular mass.

${ }^{1}$ Results represent mean \pm SD of 3 replicates.

${ }^{2}$ Deferripyoverdines of identical or very similar $( \pm \leq 1 \mathrm{Da})$ molecular masses.

The MSP that we examined were able to produce a range of different siderophores. Most of the siderophores produced contained hydroxamate functional groups, and in all of the hydroxamate-producing isolates except AFT29 and AFT36 there was correspondence with the production of pyoverdin. Production of putative phenolic siderophores by 4 isolates was detected, and only 2 of these isolates also produced pyoverdin. Two isolates were shown to produce CAS-reactive material(s) that could not be characterized as either catechol or hydroxamate. The pyoverdin-producing isolates may have been able to produce other siderophores, but these were not looked for in the pyoverdin-containing media and may not have been synthesized concurrently with the possibly more effective siderophore pyoverdin (Cornelis and Matthijs, 2002).

We were unable to characterize the siderophore(s) present in the cell-free culture supernatants of isolates

Table 2. A summary of presumed siderophore production (+) by 20 milk spoilage Pseudomonas spp.

\begin{tabular}{|c|c|c|c|c|c|}
\hline Isolate & Pyoverdin & $\begin{array}{l}\text { Unidentified } \\
\text { hydroxamate }\end{array}$ & Pyochelin & Salicylate & $\begin{array}{l}\text { Unidentified chrome } \\
\text { azurol sulfonate } \\
\text { reactive material }\end{array}$ \\
\hline B52 & + & & & & \\
\hline AFT29 & & + & & & \\
\hline AFT36 & & + & & & \\
\hline RO13 & & & + & + & \\
\hline RO93 & + & & & & \\
\hline 3 & + & & & & \\
\hline 13 & & & & & + \\
\hline 38 & + & & & & \\
\hline 65 & + & & & & \\
\hline 71 & + & & & & \\
\hline 79 & + & & + & + & \\
\hline 102 & & & & & + \\
\hline 107 & + & & & & \\
\hline 113 & + & & & & \\
\hline 117 & + & & & & \\
\hline 328 & + & & & & \\
\hline 329 & + & & + & + & \\
\hline 345 & + & & & & \\
\hline ATCC 948 & + & & & & \\
\hline ATCC 31732 & & & & + & \\
\hline Total & 14 & 2 & 3 & 4 & 2 \\
\hline
\end{tabular}


Table 3. Pyoverdin cross-feeding bioassay results, indicating those isolates that could $(+)$ or could not $(-)$ grow under growth-inhibiting irondeficient conditions when supplied with semipurified exogenous pyoverdin ${ }^{1}$

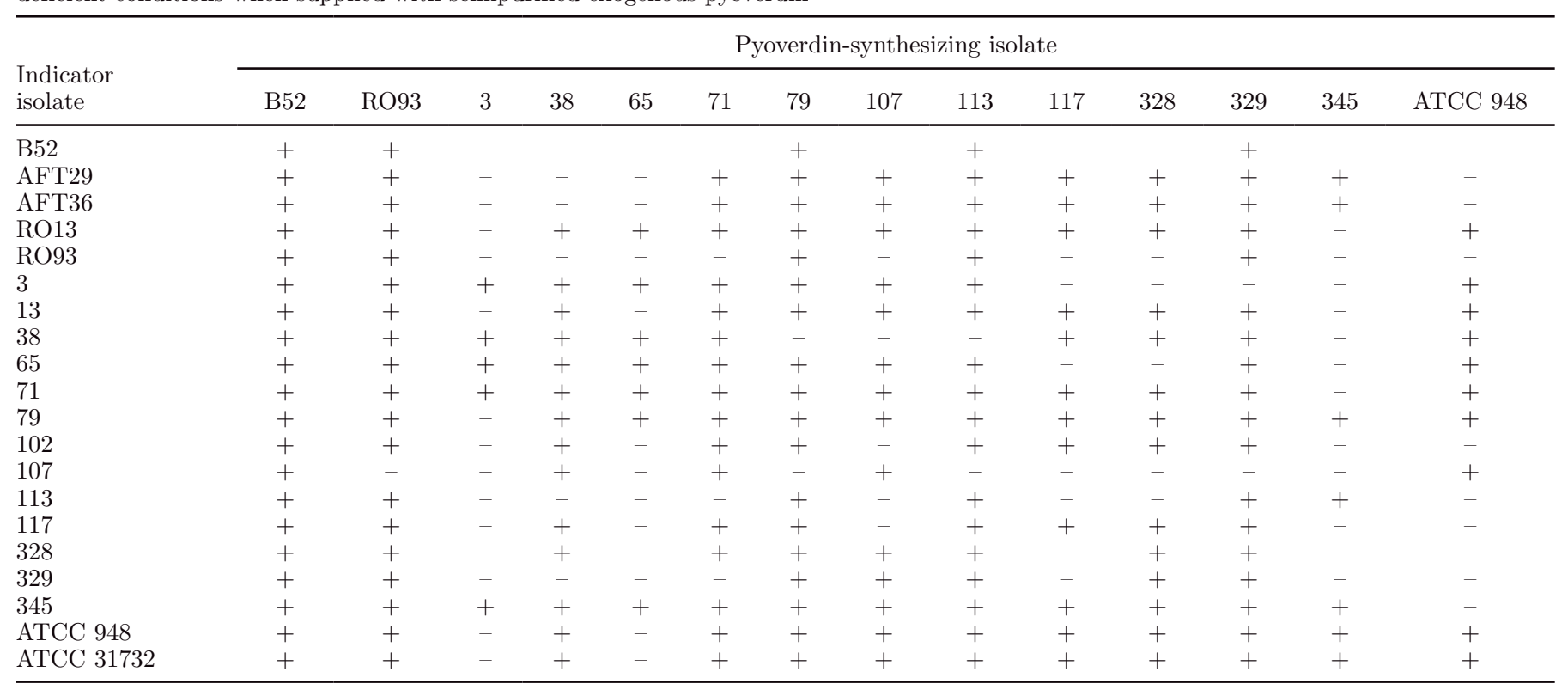

${ }^{1}$ Results are a summary of 3 replicates.

AFT29 and AFT36, but were able to determine that they differed in significant ways from other siderophores that have been associated with Pseudomonas spp. and species of related genera. The AFT29 and AFT36 siderophore(s) appeared not to contain catechol groups as are found in quinolobactin and 2,3-dihydroxy-N-benzoyl arginine. The AFT36 siderophore appeared to have a molecular mass different from those previously reported. Although material in the culture fluid of AFT36 had a molecular mass of $678 \pm 0.3$ Da, pseudomonine has a molecular mass of 330 Da; ferribactin one of $>900 \mathrm{Da}$; cepabactin one of $155 \mathrm{Da}$; aerobactin one of approximately $559 \mathrm{Da}$; ferrioxamine $\mathrm{E}$ one of 600 Da; pyridine-2,6-dithiocarboxylic acid one of $199 \mathrm{Da}$; malleobactin masses of 636, 762, and 790 Da; and the ornibactins masses of 734, 762, and 790 Da (Cornelis and Matthijs, 2002).

The ferripyoverdins produced by the 14 MSP that were tested were heterogeneous. Ten different molecular masses were detected and, according to molecular mass, only 3 of the pyoverdins were produced by more than one strain.

Cross-feeding tests showed that all of the isolates examined could use several exogenous pyoverdins produced by other MSP. The mechanisms allowing cross-feeding were not determined, but those shown by others include nonspecific receptors, broad-spectrum receptors, and multiple iron-repressible outer membrane protein receptors (Cornelis and Matthijs, 2002). The widespread presence of sequence heterogeneity and evidence of multiple receptors in 2 MSP isolates may explain both why some isolates cannot utilize a particular pyoverdin and why other isolates can utilize many pyoverdins. In the context of growth in milk, this may be a factor in why some strains may out-compete other strains. Strains that produce a siderophore with a unique stereochemistry, something that occurs among the pyoverdins because of their diverse structures, may be able to prevent other microbes from acquiring iron from their particular siderophore. By expressing multiple ferripyoverdin receptors (FPR), an isolate may at the same time be able to acquire iron from other siderophores. The ability to acquire extra iron from siderophores produced by other bacteria may be especially important during the lag phase of growth, when the total siderophore concentration is likely to be low (Bakker et al., 1990). Experiments designed to compare the growth of siderophore-deficient mutants in the presence and absence of pyoverdin-supplemented milk should provide interesting insights into this hypothesis.

The apparent similarities between the independent isolates RO93 and 79 are interesting. These isolates produced pyoverdins with apparently identical molecular weights and were able to utilize each other's pyoverdin. It is interesting that the range of pyoverdins utilized was greater for isolate 79 , possibly because of the acquisition of extra FPR determinants. Is the 1,241.7 Da pyoverdin synthesized by isolates RO93 and 79 of particular relevance to the MSP or is this simply a coincidence? 

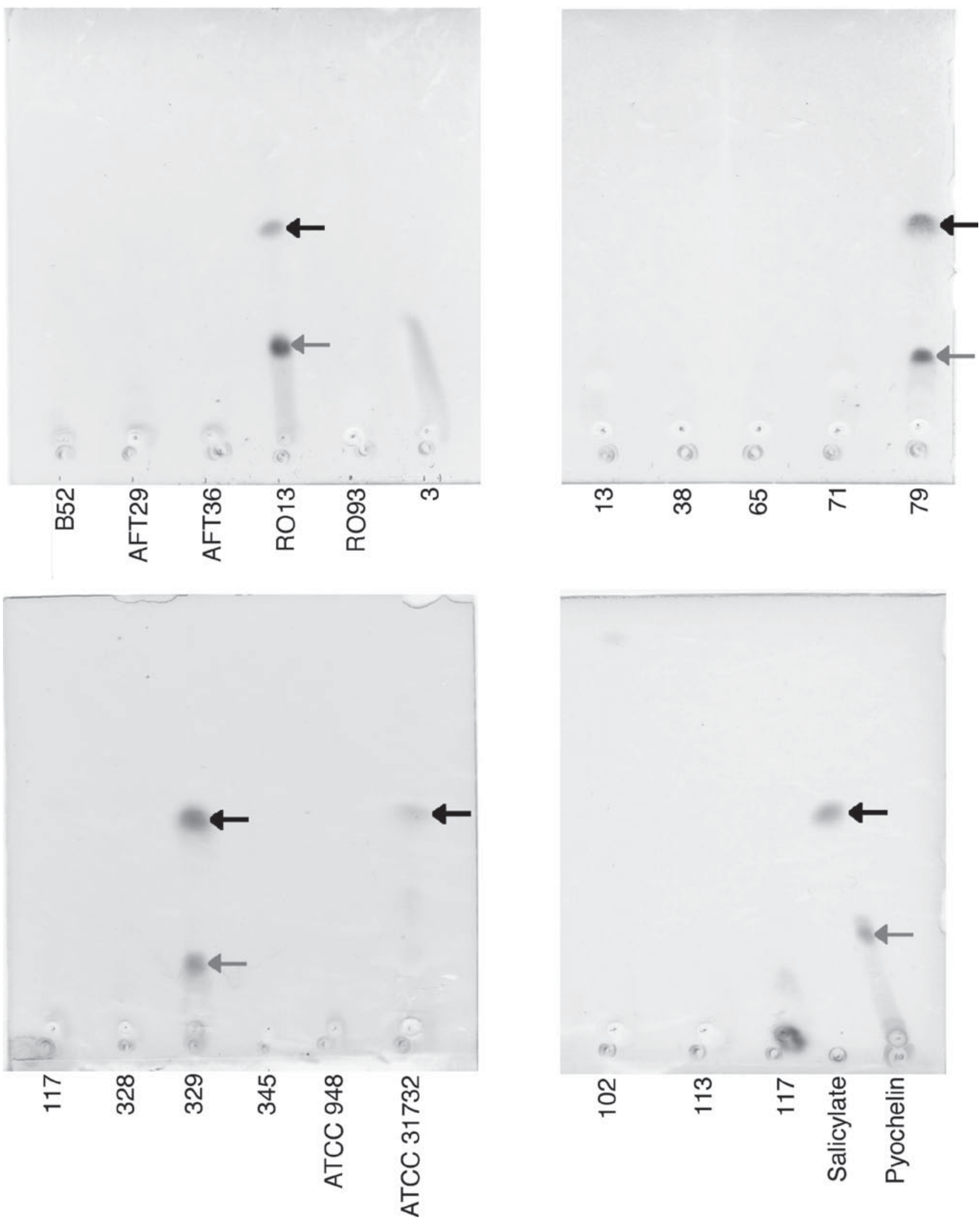

Figure 2. Thin layer chromatographs showing the separation of ethyl acetate extracts of milk spoilage Pseudomonas spp. culture supernatant after development with ferric chloride spray. The plates are $10 \mathrm{~cm}$ long in the direction of solvent migration. Black arrows indicate the presence of pyochelin and gray arrows indicate the presence of salicylate. 
The results presented here are not likely to be representative of the entire MSP population, not least because the 20 isolates studied were selected on the basis of their spoilage potential. These isolates were found to produce similar siderophores (pyoverdins) to those previously reported for saprophytic Pseudomonas spp. (de Weger et al., 1988). This is not surprising, given that most MSP entering milk are saprophytic organisms originally present in milk harvesting and milk processing environments (Duffy and Défago, 1999; Wiedmann et al., 2000).

The work reported in this paper could be usefully extended in several different ways. Future publications will describe the FPR present in the MSP studied. In addition, it would be useful to know if and when siderophores are secreted in refrigerated milk (the results reported were obtained at $25^{\circ} \mathrm{C}$ in defined medium). It is known that both growth medium and temperature may influence siderophore synthesis by Pseudomonas spp. (Garibaldi, 1971; Duffy and Défago, 1999).

Some of the siderophores detected during this work have yet to be identified. The identity of the NPHS from P. fluorescens AFT29 and AFT36 should be determined.

At the commencement of this work it was assumed that bovine milk, like human milk, is an iron-deficient environment. However, unlike human milk, bovine milk contains low levels of lactoferrin and bicarbonate, has high levels of citrate, and does not prevent the growth of pathogenic Escherichia coli (Griffiths and Humphreys, 1977), for instance. Nevertheless, the addition of iron does reduce protease and lipase production by Pseudomonas spp., probably by a mechanism involving an extracytoplasmic sigma factor, indicating that suboptimal levels of iron may be present (McKellar et al., 1987; Maunsell et al., 2006). Growth-limiting iron starvation may be achieved even in the presence of a powerful siderophore by the addition of a specific antibody raised against an important iron-repressible outer membrane receptor (Lin et al., 1998). The presence of a specific antibody blocking the relevant receptor may prevent iron from lactoferrin or other sources entering the cell even when the siderophore present can scavenge iron from lactoferrin. The complicated nature of the Pseudomonas spp. iron uptake systems may mean that more than one specific antibody may be required. Furthermore, if proteolytic and lipolytic enzyme production increases on a per cell basis due to iron starvation (McKellar et al., 1987; Fernandez et al., 1988) but cell replication is not dramatically slowed, product quality may actually be decreased. For this reason, it would be useful to know the iron status of the MSP present in milk and the effect of milk citrate, but the complex nature of milk may complicate more direct analysis. This might be done by employing iron-controlled reporter genes (Joyner and Lindow, 2000).

The widespread presence of siderophore production and cross-feeding among the MSP indicates the importance of these attributes for refrigerated milk quality. Indeed, strains possessing such attributes may have a competitive advantage in milk, and disruption of these systems, for instance with specific antibodies binding to iron-repressible outer membrane proteins, may impede the growth of bacteria involved in the spoilage of dairy products.

\section{ACKNOWLEDGMENTS}

The Dairy Research and Development Corporation (now Dairy Australia) is thanked for financial assistance. We acknowledge the assistance of Heather Craven (Food Science Australia, Werribee, Victoria, Australia), who provided the isolates studied, and that of Greg Neumann (Department of Biochemistry, La Trobe University, Victoria, Australia) with mass spectroscopy. We also thank Donald MacPhee (deceased; Department of Microbiology, La Trobe University, Victoria, Australia) for his constructive contributions to the manuscript. Nevertheless, all views expressed herein are those of the authors.

\section{REFERENCES}

Arnow, L. E. 1937. Colormetric determination of the components of 3,4-dihydroxyphenylalanine-tyrosine mixtures. J. Biol. Chem. 118:531-537.

Bakker, P. A. H. M., R. van Peer, and B. Schippers. 1990. Specificity of siderophores and siderophore receptors and biocontrol by Pseudomonas spp. Pages 131-142 in Biological Control of SoilBorne Plant Pathogens. D. Hornby, ed. CAB International, Wallingford, UK.

Bishop, J. R., and C. H. White. 1986. Assessment of dairy product quality and potential shelf-life-A review. J. Food Prot. 49:739753.

Braun, V., K. Hantke, and W. Köster. 1998. Bacterial iron transport: Mechanisms, genetics and regulation. Pages 67-145 in Iron Transport and Storage in Microorganisms, Plants, and Animals. A. Sigel and H. Sigel, ed. Marcel Dekker Inc., New York, NY.

Champagne, C. P., R. R. Laing, D. Roy, A. A. Mafu, and M. W. Griffiths. 1994. Psychrotrophs in dairy products: Their effects and their control. Crit. Rev. Food Sci. Nutr. 34:1-30.

Champomier-Vergès, M. C., A. Stintzi, and J. M. Meyer. 1996. Acquisition of iron by the non-siderophore-producing Pseudomonas fragi. Microbiology 142:1191-1199.

Cornelis, P., and S. Matthijs. 2002. Diversity of siderophore-mediated iron uptake systems in fluorescent pseudomonads: Not only pyoverdines. Environ. Microbiol. 4:787-798.

Cox, C. D., and R. Graham. 1979. Isolation of an iron-binding compound from Pseudomonas aeruginosa. J. Bacteriol. 137:357364.

Craven, H. M. 1993. Methods for the evaluation of psychrotrophic spoilage bacteria in pasteurised milk. PhD Thesis. La Trobe Univ., Melbourne, Australia.

de Weger, L. A., J. J. C. M. van Arendonk, K. Recourt, G. A. J. M. van der Hofstad, P. J. Weisbeek, and B. Lugtenberg. 1988. Siderophore-mediated uptake of $\mathrm{Fe}^{3+}$ by the plant growth- 
stimulating Pseudomonas putida strain WCS358 and by other rhizosphere microorganisms. J. Bacteriol. 170:4693-4698.

Dring, R., and P. F. Fox. 1983. Purification and characterization of a heat-stable lipase from Pseudomonas fluorescens AFT 29. Ir. J. Food Sci. Technol. 7:157-171.

Duffy, B. K., and G. Défago. 1999. Environmental factors modulating antibiotic and siderophore biosynthesis by Pseudomonas fluorescens biocontrol strains. Appl. Environ. Microbiol. 65:2429-2438.

Fernandez, L., C. San Jose, H. Cholette, and R. C. McKellar. 1988 Characterization of a pyoverdine-deficient mutant of Pseudomonas fluorescens impaired in the secretion of extracellular lipase. Arch. Microbiol. 150:523-528.

Garibaldi, J. A. 1971. Influence of temperature on the iron metabolism of a fluorescent pseudomonad. J. Bacteriol. 105:1036-1038.

Gillam, A. H., A. G. Lewis, and R. J. Andersen. 1981. Quantitative determination of hydroxamic acids. Anal. Biochem. 53:841-844.

Greenwood, K. T., and R. K. J. Luke. 1976. Studies on the enzymatic synthesis of enterochelin in Escherichia coli K-12. Four polypeptides involved in the conversion of 2,3-dihydroxybenzoate to enterochelin. Biochim. Biophys. Acta 454:285-297.

Griffiths, E., and J. Humphreys. 1977. Bacteriostatic effect of human milk and bovine colostrum on Escherichia coli: Importance of bicarbonate. Infect. Immun. 15:396-401.

Joyner, D. C., and S. E. Lindow. 2000. Heterogeneity of iron bioavailability on plants assessed with a whole-cell GFP-based bacterial biosensor. Microbiology 146:2435-2445.

Kilz, S., C. Lenz, R. Fuchs, and H. Budzikiewicz. 1999. A fast screening method for the identification of siderophores from fluorescent Pseudomonas spp. by liquid chromatography/electrospray mass spectrometry. J. Mass Spectrom. 34:281-290.

Leach, L. H., and T. A. Lewis. 2006. Identification and characterization of Pseudomonas membrane transporters necessary for utilization of the siderophore pyridine-2,6-bis(thiocarboxylic acid) (PDTC). Microbiology 152:3157-3166.

Létoffé, S., V. Redeker, and C. Wandersman. 1998. Isolation and characterization of an extracellular haem-binding protein from Pseudomonas aeruginosa that shares function and sequence similarities with the Serratia marcescens HasA haemophore. Mol. Microbiol. 28:1223-1234.

Lin, J., J. S. Hogan, and K. L. Smith. 1998. Inhibition of in vitro growth of coliform bacteria by a monoclonal antibody directed against ferric enterobactin receptor FepA. J. Dairy Sci. 81:12671274 .

Marchand, S., K. Heylen, W. Messens, K. Coudijzer, P. De Vos, K. Dewettinck, L. Herman, J. De Block, and M. Heyndrickx. 2009. Seasonal influence on heat-resistant proteolytic capacity of Pseudomonas lundensis and Pseudomonas fragi, predominant milk spoilers isolated from Belgian raw milk samples. Environ. Microbiol. 11:467-482.

Matselis, E., and I. G. Roussis. 1998. Proteinase and lipase production by Pseudomonas fluorescens. Proteolysis and lipolysis in thermized ewe's milk. Food Control 9:251-259.

Maunsell, B., C. Adams, and F. O'Gara. 2006. Complex regulation of AprA metalloprotease in Pseudomonas fluorescens M114: Evidence for the involvement of iron, the ECF sigma factor, PbrA and pseudobactin M114 siderophore. Microbiology 152:29-42.

McKellar, R. C., K. Shamsuzzaman, C. San Jose, and H. Cholette. 1987. Influence of iron(III) and pyoverdine on extracellular proteinase and lipase production by Pseudomonas fluorescens B52. Arch. Microbiol. 147:225-230.

Meyer, J. M., and M. A. Abdullah. 1978. The fluorescent pigment of Pseudomonas fluorescens: Biosynthesis, purification and physiochemical properties. J. Gen. Microbiol. 107:319-328.

Meyer, J. M., and V. A. Geoffroy. 2004. Environmental fluorescent Pseudomonas and pyoverdine diversity: How siderophores could help microbiologists in bacterial identification and taxonomy. Pages 451-468 in Iron Transport in Bacteria. J. H. Crosa, A. R. Mey, and S. M. Payne, ed. ASM Press, Washington, DC.

Meyer, J. M., A. Stintzi, V. Coulanges, S. Shivaji, J. A. Voss, K. Taraz, and H. Budzikiewicz. 1998. Siderotyping of fluorescent pseudomonads: Characterization of pyoverdines of Pseudomonas fluorescens and Pseudomonas putida strains from Antarctica. Microbiology 144:3119-3126.

Neilands, J. B. 1973. Microbial iron transport compounds (siderochromes). Pages 167-202 in Inorganic Chemistry. C. Eichhorn, ed. Elsevier Scientific Publishing Co., Amsterdam, the Netherlands.

Payne, S. M. 1994. Detection, isolation, and characterization of siderophores. Methods Enzymol. 235:329-344.

Schwyn, B., and J. B. Neilands. 1987. Universal chemical assay for the detection and determination of siderophores. Anal. Biochem. 160:47-56.

Sokol, P. A. 1986. Production and utilization of pyochelin by clinical isolates of Pseudomonas cepacia. J. Clin. Microbiol. 23:560-562.

Stepaniak, L., P. F. Fox, and C. Daly. 1982. Isolation and general characterization of a heat-stable proteinase from Pseudomonas fluorescens AFT 36. Biochim. Biophys. Acta 717:376-383.

Suhren, G. 1989. Producer microorganisms. Pages 3-34 in Enzymes of Psychrotrophs in Raw Food. R. C. McKellar, ed. CRC Press, Boca Raton, FL.

Visca, P., A. Ciervo, V. Sanfilippo, and N. Orsi. 1993. Iron-regulated salicylate synthesis by Pseudomonas spp. J. Gen. Microbiol. 139:1995-2001.

Visca, P., G. Colotti, L. Serino, D. Verzili, N. Orsi, and E. Chiancone. 1992. Metal regulation of siderophore synthesis in Pseudomonas aeruginosa and functional effects of siderophore-metal complexes. Appl. Environ. Microbiol. 58:2886-2893.

Wiedmann, M., D. Weilmeier, S. S. Dineen, R. Ralyea, and K. J. Boor. 2000. Molecular and phenotypic characterization of Pseudomonas spp. isolated from milk. Appl. Environ. Microbiol. 66:20852095.

Woods, R. G., M. Burger, C. A. Beven, and I. R. Beacham. 2001. The aprX-lipA operon of Pseudomonas fluorescens B52: A molecular analysis of metalloprotease and lipase production. Microbiology $147: 345-354$ 